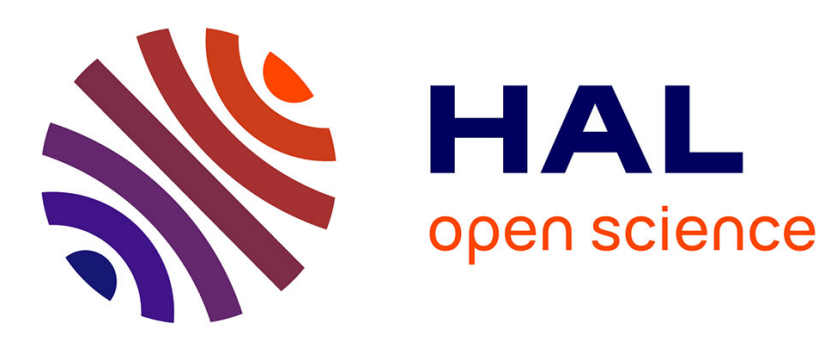

\title{
A Domain Decomposition Method for the Helmholtz Equation and Related Optimal Control Problems
}

\author{
Jean-David Benamou, Bruno Desprès
}

\section{To cite this version:}

Jean-David Benamou, Bruno Desprès. A Domain Decomposition Method for the Helmholtz Equation and Related Optimal Control Problems. [Research Report] RR-2791, INRIA. 1996. inria-00073899

\section{HAL Id: inria-00073899 \\ https://hal.inria.fr/inria-00073899}

Submitted on 24 May 2006

HAL is a multi-disciplinary open access archive for the deposit and dissemination of scientific research documents, whether they are published or not. The documents may come from teaching and research institutions in France or abroad, or from public or private research centers.
L'archive ouverte pluridisciplinaire HAL, est destinée au dépôt et à la diffusion de documents scientifiques de niveau recherche, publiés ou non, émanant des établissements d'enseignement et de recherche français ou étrangers, des laboratoires publics ou privés. 


\section{A Domain Decomposition Method for the Helmholtz equation and related Optimal Control Problems}

Jean-David Benamou , Bruno DesprŁs

\section{N 2791}

Fevrier 1996

PROGRAMME 6

apport

de recherche 



\title{
A Domain Decomposition Method for the Helmholtz equation and related Optimal Control Problems
}

\author{
Jean-David Benamou * , Bruno Desprès ** \\ Programme 6 - Calcul scientifique, modélisation et logiciel numérique \\ Projet Ident
}

Rapport de recherche $\mathrm{n}^{\circ} 2791$ - Fevrier 1996 - 30 pages

\begin{abstract}
We present an iterative domain decomposition method to solve the Helmholtz equation and related optimal control problems. The proof of convergence of this method relies on energy techniques. This method leads to efficient algorithms for the numerical resolution of harmonic wave propagation problems in heterogeneous media and their control. Keywords : Domain decomposition, Helmholtz equation, Harmonic waves, Optimal control, Waveguide, Absorbing boundary conditions
\end{abstract}

AMS(MOS) subject classification : $35 \mathrm{~J} 05,49 \mathrm{M} 99,65 \mathrm{~N} 55$

(Résumé : tsvp)

*INRIA, Domaine de Voluceau, B.P.105 78153 Le Chesnay cedex, France, e-mail :benamou@misstick.inria.fr

${ }^{* *}$ Commissariat à l'Energie Atomique, 94250 Villeneuve Saint Georges cedex, France, email :despres@limeil.cea.fr 


\section{Une méthode de décomposition de domaine pour l'équation de Hemlholtz et les problèmes de contrôle optimal associés}

Résumé : Nous présontons une méthode de décomposition de domaine itérative pour résoudre l'équation de Helmholtz et les problèmes de contrôles optimal qui lui sont associés. La preuve de la convergence de cette méthode utilise des estimations d'énergies. Cette méthode produit des algorithmes efficaces pour la résolution numérique de problèmes de propagations d'ondes en régime harmonique en milieu inhomogènes et leur contrôle.

Mots-Clés : Décomposition de Domaine, Equation de Hemlholtz, Ondes Harmoniques, Contrôle Optimal, Guides d'Ondes, Conditions Absorbantes 


\section{Introduction}

The numerical resolution of the Helmholtz equation and related optimal control problem in heterogeneous media at high wave number is a challenging problem. This model finds applications in electromagnetic and acoustic wave propagation. Because of the oscillatory character of the solution, the necessary fineness of discretization (at least ten points per wavelength, also known as 'rule of thumb') leads at high wave numbers to very large non hermitian (possibly non-symmetric) complex linear systems exceeding the current computer memory capabilities. It therefore rules out direct methods to solve the direct problem. It makes the resolution of optimal control problems for which gradient type methods need to solve a sequence of such direct equation and similar adjoint equations even harder.

Integral methods [13] [37] [30] or fictitious domain methods [1] [7] do not apply to heterogeneous media. Iterative methods such as preconditioned GMRES [36] or bi-conjugate gradient [12] have hieratic numerical convergence and there is actually no theoretical proof of convergence. A domain decomposition for non-symmetric and indefinite problems is also discussed in [9] [10]. For very high frequency, asymptotic methods is an efficient solution [8] but still of delicate use. There is nevertheless a need for the resolution of the Helmholtz equation under their range of validity.

The present domain decomposition method is a general and efficient solution. It moreover extends to the numerical resolution of optimal control problems for systems governed by the Helmholtz equation. Solving such a problem classically require to iterate the resolution of direct and adjoint Helmholtz problems in order to compute descent directions for a gradienttype method.

The idea is to split the domain into smaller subdomains and solve a sequence of similar subproblems on these subdomains. The boundary conditions are adjusted iteratively by ad-hoc transmission conditions between adjacent subdomains. The number and size of subdomains can now be chosen so as to enable direct methods to solve the subproblems. In the case of optimal control, we decompose the coupled system made of the direct and adjoint Helmholtz equation and the optimality condition which variationally express that the control is optimal. This method actually solves at the same time the equations and the optimization problem whereas classical methods require to iterate the resolution of direct and adjoint problems in order to compute descent directions for a gradient-type method.

The method is easily implemented and naturally adapted to parallel computers, which use is a major trend in modern scientific computing.

The aim of this paper is to give in a unified framework a formal presentation of the algorithms and the energy estimates that lead to the proofs of convergence. Comprehensive mathematical studies can be found in [17] [16] [15] [3].

We focus on these energy estimates since:

i) These estimates are not standard in the context of elliptic coercive problems.

ii) They help to understand why the algorithms converge.

iii) Slight modifications of this technique can be used in various cases of boundary conditions and equations.

$\mathrm{RR} \mathbf{n}^{\circ} 2791$ 
We will use a continuous formulation of the equations throughout the paper. The domain decomposition method can also be applied to the discretized equations with corresponding energy estimates and convergence proofs (see references in section 6).

We present in section 2 the domain decomposition method for the resolution of the Helmholtz equation. A proof of convergence is given based on energy estimates. Section 3 deals with the optimal control of systems governed by such equations. Different 'coupled' transmission conditions are introduced. Convergence is again obtained using energy estimates but the arguments differ from section 2. We present in section 4 two variants of this algorithm. One is based on a relaxation of the transmission condition, the other uses the optimal control transmission conditions to solve the direct problem. Section 5 discuss the application of the domain decomposition and its variants presented in section 4 to the inhomogeneous case and various boundary condition. A test case is solved numerically in section 6 where we briefly discuss numerical issues.

\section{DDM for the Helmholtz problem}

\subsection{The Helmholtz equation and the truncated domain}

Let $\Omega$ be a sufficiently smooth bounded open set in ${ }^{2}$ or ${ }^{3}$ containing an obstacle. The boundary $\Gamma=\partial \Omega$ is divided into an interior boundary $\Gamma_{\text {int }}$ (the boundary of the obstacle) and an exterior boundary $\Gamma_{e x t}$ (see fig 1 for example).

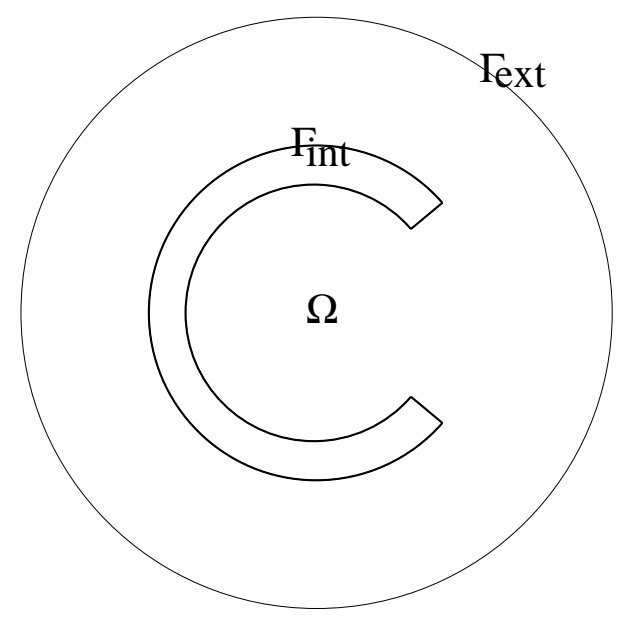

Figure 1: geometry of the diffraction problem: the resonator obstacle 
The out-going normal is denoted by $\nu$. In a scattering problem the source term $u_{\text {inc }}$, called the incident wave (usually a plane wave), illuminates the object located inside $\Gamma_{\text {int }}$. The scattered wave is the complex solution of :

$$
\left\{\begin{array}{lr}
\left(-\nabla(\mu \vec{\nabla} u)-\omega^{2} \rho u=f\right. & \text { in } \Omega \\
\frac{\partial}{\partial \nu} u=-\frac{\partial}{\partial \nu} u_{i n c} & \text { on } \Gamma_{i n t} \\
\frac{\partial}{\partial \nu} u+i \omega \sqrt{\frac{\rho}{\mu}} u=0 & \text { on } \Gamma_{\text {ext }},
\end{array}\right.
$$

where $\omega$ is the frequency of the harmonic oscillations. The coefficients $\mu$ and $\rho$ are strictly positive bounded, possibly discontinuous, real functions characterizing the non dispersive medium. Their physical interpretation vary according the modeled physical situation [37]. The source term $f$ is given and arise from these inhomogeneities.

The boundary condition on $\Gamma_{e x t}$ is an absorbing boundary condition, of the first order (following [2]). It approximates the outgoing character of the scattered wave on the truncated domain. In the exact model, the solution is defined in all space and satisfies the Sommerfeld radiation condition (expressed in polar coordinates, $r$ is the radius):

$$
\frac{\partial}{\partial r} u+i \omega \sqrt{\frac{\rho}{\mu}} u=O\left(\frac{1}{r^{2}}\right) \text { when } r \text { goes to }+\infty .
$$

This approximate boundary condition is introduced to bound the domain for actual computations. It is important to notice that the first order boundary condition on $\Gamma_{e x t}$ plays a fundamental role in the well posedness (i.e. existence and uniqueness of a solution) of (1) (see [24] [17]).

The Neumann boundary condition imposed on $\Gamma_{i n t}$ simulates the presence of a 'hard' object. If one ever wants to study the scattering by a 'soft' object, one has to use a Dirichlet boundary condition $u=-u_{\text {inc }}$. Impedance boundary conditions :

$$
\frac{\partial}{\partial \nu} u+i \omega z u=-\frac{\partial}{\partial \nu} u_{i n c}+i \omega z u_{i n c}
$$

where $z$ is a complex number are also possible. $\operatorname{Re}(z) \geq 0$ is a necessary condition to obtain well posedness. This is a compatibility condition with the first order absorbing boundary condition. Impedance boundary condition can be derived from the Leontovitch boundary condition [8] for electromagnetic waves.

Higher order boundary conditions for $\Gamma_{\text {ext }}$ exist [2] [23], which lead to better approximations of the transparency nature of the exterior boundary. For example a second order boundary condition is given by :

$$
\frac{\partial}{\partial \nu} u+i \omega\left(u+\frac{1}{2 \omega^{2}} \Delta \text { tangential } u\right)=0 .
$$

One may also want to use non-local boundary conditions on $\Gamma_{e x t}$. For waveguide computations, for example, an exact outgoing boundary condition can be derived at the exit of

$\operatorname{RR} n^{\circ} 2791$ 
the waveguide by mean of a modal decomposition. The boundary condition now takes the form

$$
\frac{\partial}{\partial \nu} u+T(u)=0
$$

where $\mathrm{T}$ is an integral operator which involves sine or cosine transforms on $\Gamma_{e x t}$ which represents here the exit of the wave guide [32].

Finally let us mention the recent Perfectly Matched Layer technique [6] which consists in adding an absorbing layers around the computational domain. These PML are remarkable because they generate (for the continuous equations) no artificial reflections at the interface between the domain and the layers. Their numerical discretization and implementation for the Helmholtz equation have been studied with good results in [6] [34]. We briefly describe these PML in a simplified situation. We consider the homogeneous (i.e. $\mu=\rho=1, f=0$ ) case in ${ }^{2}$. Let $(x, y)$ denote the space coordinates. Suppose we want to solve the problem in the half space $x<0$. We extend the problem to the union of the half plane and an absorbing layer defined as a strip $0<x<\delta$. We solve in this added layer the modified equation :

$$
-\partial_{x x}^{2} u-d \partial_{y}\left(d \partial_{y} u\right)-\omega^{2} u=0
$$

where $d=\frac{i \omega}{i \omega+\sigma}$ and $\sigma$ is a real parameter which is responsible for the absorption. The sign of $\sigma$ determines the in-going/out-going character of the scattered wave.

\subsection{The Domain Decomposition Method}

We simplify the description of the method by restraining ourselves to the homogeneous case (i.e. $\mu=\rho=1, f=0$ ). The extension to the heterogeneous case is discussed in section 4.2. The equation is now

$$
\left\{\begin{array}{lr}
-\Delta u-\omega^{2} u=0 & \text { in } \Omega \\
\frac{\partial}{\partial \nu} u=-\frac{\partial}{\partial \nu} u_{i n c} & \text { on } \Gamma_{i n t} \\
\frac{\partial}{\partial \nu} u+i \omega u=0 & \text { on } \Gamma_{e x t}
\end{array}\right.
$$

\subsubsection{Transmission conditions}

Let us consider a trivial case where $\Omega$ is split into 2 sub-domains $\Omega_{1}$ and $\Omega_{2}$ such that the frontier of $\Omega_{2}$ has an empty intersection with $\partial \Omega$ (see fig 2).

We denote by $u_{1}$ and $u_{2}$ the restrictions of $u$ (solution of (7)) in respectively $\Omega_{1}$ and $\Omega_{2}$. $u_{1}$ and $u_{2}$ satisfy the following interface conditions on $\partial \Omega_{1} \cap \partial \Omega_{2}$ :

$$
\begin{aligned}
u_{1} & =u_{2} \\
\frac{\partial}{\partial \nu_{1}} u_{1} & =-\frac{\partial}{\partial \nu_{2}} u_{2}
\end{aligned}
$$

where $\nu_{i}$ is the exterior normal to $\Omega_{i}$. If $\frac{\partial}{\partial \nu_{2}} u_{2}$ is given, $u_{1}$ satisfy the equation : $-\Delta u_{1}-\omega^{2} u_{1}=0$, with the boundary conditions (9) above. 


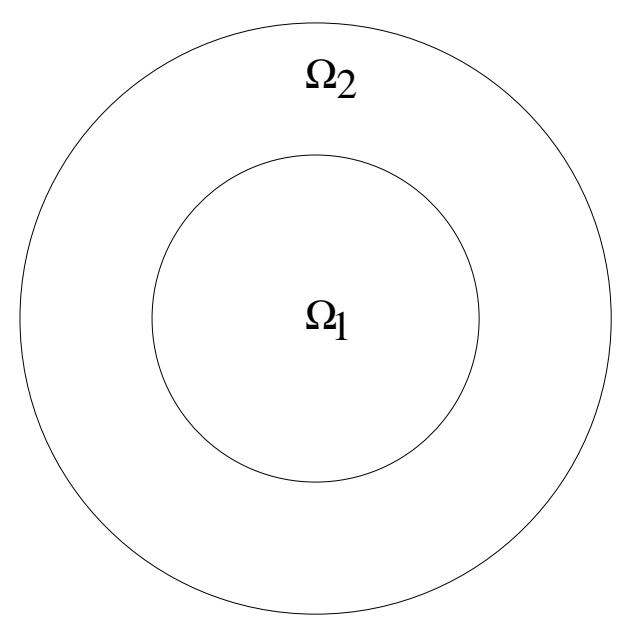

Figure 2: simple decomposition

It is well known however that this problem may be ill-posed due to the existence of eigenvalues of the Laplace operator [33]. We propose instead to combine linearly equations (8) and (9) to get the following equivalent boundary conditions :

$$
\frac{\partial}{\partial \nu_{2}} u_{2}+i \omega u_{2}=-\frac{\partial}{\partial \nu_{1}} u_{1}+i \omega u_{1}
$$

and

$$
\frac{\partial}{\partial \nu_{1}} u_{1}+i \omega u_{1}=-\frac{\partial}{\partial \nu_{2}} u_{2}+i \omega u_{2} .
$$

The sub-problem on $\Omega_{1}$ together with condition (11) is now well posed. This sub-problem is of the same type as the global problem which is well-posed thanks to the absorbing boundary condition (see above). These mixed (or 'Robin') boundary conditions play an important role in the definition of our domain decomposition method.

\subsubsection{The basic algorithm}

We describe the domain decomposition algorithm. The idea is to adjust iteratively the boundary conditions at the interfaces between subdomains to obtain the transmission conditions of the type (10) (11).

We introduce some notations. Let us split $\Omega$ into a finite number of non-overlapping sub-domains $\Omega_{k}, 1 \leq k \leq K$, with sufficiently smooth boundaries. These sub-domains have interfaces denoted by $\Sigma_{k j}=\Sigma_{j k}=\partial \Omega_{k} \cap \partial \Omega_{j}$. They may also have a part of their boundaries impinging on $\Gamma$. So we write $\Gamma_{k, e x t}=\partial \Omega_{k} \cap \Gamma_{e x t}$ and $\Gamma_{k, \text { int }}=\partial \Omega_{k} \cap \Gamma_{\text {int }}$ (see figure 3 ). The out-going normal for $\Omega_{k}$ is $\nu_{k}$. 


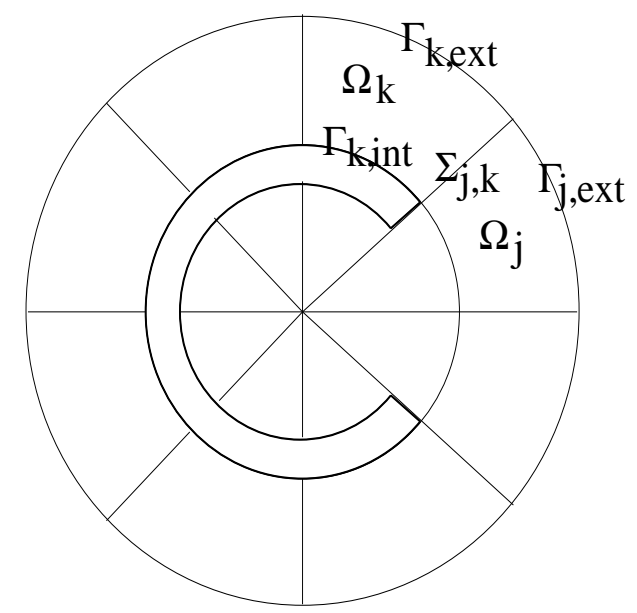

Figure 3: A decomposition of the domain

We now define the following iterative procedure using Robin transmission conditions (the superscript denotes the rank of the iterative procedure)

Initialize $u_{k}^{0}$ for all $k$, then, iterate for $n>0$ :

$$
\left\{\begin{array}{lr}
-\Delta u_{k}^{n+1}-\omega^{2} u_{k}^{n+1}=0 & \text { in } \Omega_{k} \\
\frac{\partial}{\partial \nu_{k}} u_{k}^{n+1}+i \omega u_{k}^{n+1}=-\frac{\partial}{\partial \nu_{j}} u_{j}^{n}+i \omega u_{j}^{n} & \text { on } \Sigma_{k j} \\
\frac{\partial}{\partial \nu_{k}} u_{k}^{n+1}+i \omega u_{k}^{n+1}=0 & \text { on } \Gamma_{k, \text { ext }} \\
\frac{\partial}{\partial \nu_{k}} u_{k}^{n+1}=\frac{\partial}{\partial \nu_{k}} u_{i n c} & \text { on } \Gamma_{k, \text { int }} .
\end{array}\right.
$$

The boundary condition on $\Gamma_{k, \text { int }}$ for the subproblems is determined according the boundary condition on $\Gamma_{\text {int }}$ of the global problem (7) (i.e. $u_{k}^{n+1}=u_{i n c}$ on $\Gamma_{k, \text { int }}$ for a soft obstacle and so forth ...). When $\Gamma_{k, \text { int }}=\emptyset$ or $\Gamma_{k, e x t}=\emptyset$,i.e. $\Omega_{k}$ is an 'interior' subdomain, the corresponding boundary condition is simply ignored. This algorithm is an Helmholtz adaptation of the well known Schwarz algorithm for elliptic problems described in [28]. Thanks to the Robin transmission conditions, the sub-problems are well posed. Notice that, at each step of the iterative procedure, the resolution of each subproblem is explicit and independent of the other subproblems.

\subsubsection{Convergence}

We are able to prove the convergence of the procedure (12) under various hypothesis on the regularity of the solution [17]. We do not want to go inside the mathematical details of the proof. We instead assume enough regularity on the global solution of (7) and of the 
initialization $\left(u_{k}^{0}\right)$ of the iterative procedure to be able to define a 'pseudo-energy' linked with the algorithm. Let us define the error

$$
e u_{k}^{n}=u_{k}^{n}-u
$$

It satisfies equations (12) with $u_{i n c}=0$. The "pseudo-energy" at iteration $n$ has the form (|.| is the complex modulus):

$$
E^{n}=\sum_{k \neq j} \int_{\Sigma_{k j}}\left(\left|\frac{\partial}{\partial \nu_{k}} e u_{k}^{n}\right|^{2}+\omega^{2}\left|e u_{k}^{n}\right|^{2}\right) d \sigma
$$

We call that quantity a 'pseudo-energy' because it is not a conventional energy. However, if $E^{n}=0$, then both $e u_{k}^{n}$ and $\frac{\partial}{\partial \nu_{k}} e u_{k}^{n}$ are equal to 0 on $\Sigma_{k j}$. This implies that (see [13]) $e u_{k}^{n}=0$ in $\Omega_{k}$. have:

The domain decomposition algorithm turns out to decrease this 'pseudo-energy'. We

Proposition 1 The "pseudo-energy" satisfies

$$
E^{n+1}=E^{n}-2 \omega^{2} \sum_{k} \int_{\Gamma_{k, e x t}}\left|e u_{k}^{n+1}\right|^{2}+\left|e u_{k}^{n}\right|^{2} d \sigma
$$

The proof comes from the following computations. First we see that

$$
\begin{aligned}
E^{n+1}= & \sum_{k \neq j} \int_{\Sigma_{k j}}\left|\frac{\partial}{\partial \nu_{k}} e u_{k}^{n+1}+i \omega e u_{k}^{n+1}\right|^{2} d \sigma- \\
& 2 \omega^{2} \sum_{k} \int_{\partial \Gamma_{k, \text { ext }}}\left|e u_{k}^{n+1}\right|^{2} d \sigma .
\end{aligned}
$$

Indeed, using the equation on each subdomain and integrating by part against the conjugate of $i \omega e u_{k}^{n+1}$, we have :

$$
\begin{gathered}
\Re e\left(\int_{\partial \Omega_{k}} \frac{\partial}{\partial \nu_{k}} e u_{k}^{n+1} \overline{i \omega e u_{k}^{n+1}} d \sigma\right) \\
=\Re e\left(-i \omega\left(\int_{\Omega_{k}}\left|\nabla e u_{k}^{n+1}\right|^{2}-\omega^{2}\left|e u_{k}^{n+1}\right|^{2}\right) d x\right)=0
\end{gathered}
$$

Then, using the boundary conditions on $\Gamma_{k, \text { int }}, \Gamma_{k, e x t}$, we recombine in (14) the integral on the the boundary of each subdomain to express the missing cross products in (15).

Using the transmission conditions we get :

$$
\begin{aligned}
E^{n+1}= & \sum_{k \neq j} \int_{\Sigma_{k j}}\left|-\frac{\partial}{\partial \nu_{k}} e u_{k}^{n}+i \omega e u_{k}^{n}\right|^{2} d \sigma- \\
& 2 \omega^{2} \sum_{k} \int_{\partial \Gamma_{k, \text { ext }}}\left|e u_{k}^{n+1}\right|^{2} d \sigma .
\end{aligned}
$$

$\operatorname{RR} n^{\circ} 2791$ 
We finally use the analog of (16) at step $n$ to obtain (14).

We deduce from proposition 1 that :

i) $\left(E^{n}\right)$ is a bounded sequence.

ii) $\sum_{k} \int_{\Gamma_{k, \text { ext }}}\left|e u_{k}^{n}\right|^{2} d \sigma$ goes to 0 as the generic term of a convergent serie.

This is enough to prove the convergence of the domain decomposition method. We establish that the error is null in the subdomain bordering $\Gamma_{e x t}$ and show the same property for the interior subdomain by an iterative progression.

The convergence theorem is (see [17]) :

Theorem 1 For all $k$, we have

$$
\int_{\Omega_{k}}\left|\nabla u_{k}^{n}-\nabla u\right|^{2}+\left|u_{k}^{n}-u\right|^{2} \text { dx goes to } 0 \text { with } n
$$

We want here to point out the importance of the first order absorbing condition in the convergence process. It is used in the proof to obtain the quantity $\sum_{k} \int_{\Gamma_{k}, \text { ext }}\left|e u_{k}^{n}\right|^{2} d \sigma$ in the right hand side of (14), which decreases the pseudo-energy of the error.

An impedance boundary condition on $\Gamma_{\text {int }}(3)$ will turn this term into $-\Re e(z) \int_{\Gamma_{k, \text { int }}}\left|e u_{k}^{n}\right|^{2} d \sigma$. Hence the importance of the sign of $z$ stressed in section 2.1.

An other choice of boundary condition on $\Gamma_{e x t}$ such as a second order absorbing boundary condition (4) or a non-local operator $T$ as for wave guides (5) does not allow to prove convergence of the algorithm. This point is discussed in section 5.2.

\section{DDM for the optimal control}

\subsection{The optimal control problem}

We consider the problem of the optimal control of a system governed by the Helmholtz problem (7) of section 3.

The boundary $\Gamma_{\text {int }}$ of the scattering obstacle is now split in two parts (see figure 4).

One part on which we have the previous scattering boundary condition and still denoted $\Gamma_{\text {int }}$. A second part called $\Gamma_{c t r}$ (between the dots on figure 4) on which we add a control variable $v$, complex valued function, in the boundary condition :

$$
\frac{\partial}{\partial \nu} u=-\frac{\partial}{\partial \nu} u_{i n c}+v \text { on } \Gamma_{c t r}
$$

The solution $u(v)$ of the scattering problem (7) (18) now depends on the control $v$ which models artificial emission of surface currents for electro-magnetic waves or forced vibration for acoustic waves.

We want to solve the optimization problem:

$$
\min _{v \in U} J(u(v), v)
$$




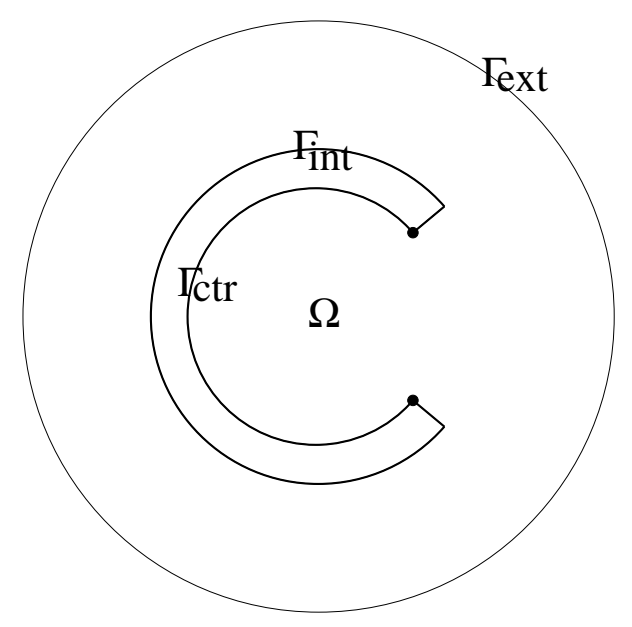

Figure 4: New decomposition of the boundary of the scatterer

where $U$ is a closed convex set of admissible controls and $\mathbf{J}$ a given cost functional.

The simplest example of such a cost function is given by

$$
J(u, v)=\left\{\int_{\Omega} \frac{1}{2}|u|^{2} d x+\frac{\alpha}{2} \int_{\Gamma_{c t r}}|v|^{2} d \sigma\right\}
$$

$\alpha$ is a strict positive penalization parameter. The first part of the above functional is a norm of the scattered field. We try to make the scatterer invisible to the probing incident plane wave. The penalization part takes into account the norm of the control. Adjusting the penalization parameter $\alpha$, we can make a compromise between the minimization of the energy of the scattered field and the cost of this minimization. As we only control a part of the boundary of the scattering object and because of the penalization term, the solution is not trivially $v=-u_{\text {inc }}$.

Thanks to the strict convexity of the functional, the optimization problem has a unique solution. It is characterized by the following adjoint problem :

$$
\left\{\begin{array}{lr}
-\Delta p-\omega^{2} p=u & \text { in } \Omega \\
\frac{\partial}{\partial y} p=0 & \text { on } \Gamma_{\text {int }} \cup \Gamma_{c t r} \\
\frac{\partial}{\partial \nu} p-i \omega p=0 & \text { on } \Gamma_{\text {ext }}
\end{array}\right.
$$

and optimality condition :

$$
\int_{\Gamma_{c t r}} \Re e((p+\alpha v)(\overline{w-v})) d \sigma \geq 0, \quad \forall w \in U,
$$

which provide a variational expression of the gradient of the functional. For more general formulations of optimal control problems see [27]. 


\subsection{The Domain Decomposition Method}

We present in this section the application of the domain decomposition algorithm to the resolution of the coupled system (7) (18) (21) (22).

\subsubsection{The iterative algorithm}

The domain $\Omega$ is decomposed as in section 2.2. We keep the same notations and add $\Gamma_{k, c t r}=\partial \Omega_{k} \cap \Gamma_{c t r}$.

We define a family $\left(U_{k}\right)$ of closed convex sets of admissible local controls on each $\Gamma_{k, c t r}$ satisfying compatibility conditions with $U$ :

$$
\begin{aligned}
& \forall v \in U,\left.v\right|_{\Gamma_{k, c t r}} \in U_{k} \text { and } \\
& \forall v_{k} \in U_{k}, \quad v \text {, such that }\left.v\right|_{\Gamma_{k, c t r}}=v_{k} \forall k, \text { belongs to } U .
\end{aligned}
$$

These conditions are satisfied by the usual local constraints on the control variable. A typical example is :

$$
\begin{aligned}
& U=\left\{v, \xi_{0}(x) \leq v(x) \leq \xi_{1}(x) \text { for a.e. } x \in \Gamma_{c t r}\right\} \text { and } \\
& U_{k}=\left\{v_{k}, \xi_{0}(x) \leq v_{k}(x) \leq \xi_{1}(x) \text { for a.e. } x \in \Gamma_{k, c t r}\right\} \\
& \xi_{0}, \xi_{1} \in L^{\infty}\left(\Gamma_{c t r}\right)
\end{aligned}
$$

Conversely, global or state constraints do not a priori satisfy (23).

We now describe the method, as in section 2.2 ,

Initialize $u_{k}^{0}, p_{k}^{0}$ for all $k$, then iterate for $n>0$ :

$$
\begin{gathered}
\left\{\begin{array}{lc}
-\Delta u_{k}^{n+1}-\omega^{2} u_{k}^{n+1}=0 & \text { in } \Omega_{k} \\
\frac{\partial}{\partial \nu_{k}} u_{k}^{n+1}+i \omega u_{k}^{n+1}=0 & \text { on } \Gamma_{k, \text { ext }} \\
\frac{\partial}{\partial \nu_{k}} u_{k}^{n+1}=\frac{\partial}{\partial \nu_{k}} u_{i n c} & \text { on } \Gamma_{k, \text { int }} \\
\frac{\partial}{\partial \nu_{k}} u_{k}^{n+1}=\frac{\partial}{\partial \nu_{k}} u_{i n c}+v_{k}^{n+1} & \text { on } \Gamma_{k, c t r}
\end{array}\right. \\
\left\{\begin{array}{lr}
-\Delta p_{k}^{n+1}-\omega^{2} p_{k}^{n+1}=u_{k}^{n+1} & \text { in } \Omega_{k} \\
\frac{\partial}{\partial \nu_{k}} p_{k}^{n+1}-i \omega p_{k}^{n+1}=0 & \text { on } \Gamma_{k, \text { ext }} \\
\frac{\partial}{\partial \nu_{k}} p_{k}^{n+1}=0 & \Gamma_{k, \text { int }} \cup \Gamma_{k, c t r}
\end{array}\right. \\
\int_{\Gamma_{k, \text { ctr }}} \Re\left(\left(p_{k}^{n+1}+\alpha v_{k}^{n+1}\right)\left(\overline{\left.w_{k}-v_{k}^{n+1}\right)}\right) d \sigma \geq 0, \forall w_{k} \in U_{k} .\right.
\end{gathered}
$$

These subproblems are simply the restriction of our original problem to the subdomains. The unknowns are $u_{k}^{n+1}, p_{k}^{n+1}$ and a local control variable $v_{k}^{n+1}$ for the subdomains bordering the control boundary, i.e. such that $\Gamma_{k, c t r} \neq \emptyset$.

We need to specify the transmission conditions on the interfaces $\Sigma_{k j}$ between subdomains in order to ensure that the sequence of local solutions of these subproblems converges to the solution of the global problem. 


\subsubsection{The transmission conditions}

For the Helmholtz equation we used transmissions conditions in the form of Robin boundary conditions :

$$
\frac{\partial}{\partial \nu_{k}} u_{k}^{n+1}+i \omega u_{k}^{n+1}=-\frac{\partial}{\partial \nu_{j}} u_{j}^{n}+i \omega u_{j}^{n}
$$

If we try to apply the same transmission conditions to the direct and adjoint equation which are both Helmholtz equations, i.e. :

$$
\frac{\partial}{\partial \nu_{k}} p_{k}^{n+1}+i \omega p_{k}^{n+1}=-\frac{\partial}{\partial \nu_{j}} p_{j}^{n}+i \omega p_{j}^{n}
$$

we cannot prove the convergence of the algorithm using the energy technique of section 2.3. The reason beeing the coupling between the direct, adjoint and control variable in the equations.

This same coupling suggested the introduction of coupled transmission conditions in [3] for the optimal control of systems governed by scalar elliptic equations. We show here that this is also a suitable choice in the case of systems governed by the Helmholtz equation. The coupled transmission conditions take the form:

$$
\begin{aligned}
& \frac{\partial}{\partial \nu_{k}} u_{k}^{n+1}+\lambda p_{k}^{n+1}=-\frac{\partial}{\partial \nu_{j}} u_{j}^{n}+\lambda p_{j}^{n} \text { on } \Sigma_{k j}, \\
& \frac{\partial}{\partial \nu_{k}} p_{k}^{n+1}-\lambda u_{k}^{n+1}=-\frac{\partial}{\partial \nu_{j}} p_{j}^{n}-\lambda u_{j}^{n} \text { on } \sigma_{k j},
\end{aligned}
$$

with $\lambda$ a real positive parameter.

\subsubsection{Decomposition in local optimal control problems}

The algorithm (24) (25) (27) (26) is now well defined, i.e. it can be shown that the subproblems are well posed. They can actually be reinterpreted as local optimal control problems.

Let us define the local functional

$$
\begin{aligned}
J_{k}\left(\tilde{v}_{k}\right)= & \int_{\Omega_{k}} \frac{1}{2}\left|\tilde{u}_{k}\right|^{2} d x+\int_{\Gamma_{k, c t r}} \frac{\alpha}{2}|\tilde{v}|^{2} d \sigma+ \\
& \sum_{j} \int_{\Sigma_{k j}} \frac{\lambda}{2}\left(\left|\frac{\partial}{\partial \nu_{k}} \tilde{p}_{k}\right|^{2}+\left|\tilde{p}_{k}\right|^{2}\right) d \sigma .
\end{aligned}
$$

where $\tilde{u}_{k}, \tilde{p}_{k}$ are functions of $\tilde{v}_{k}$ and solution of the coupled subproblems (24) (25) (27) with $\tilde{u}_{k}, \tilde{p}_{k}, \tilde{v}_{k}$ instead of $u_{k}^{n+1}, p_{k}^{n+1}, v_{k}^{n+1}$. This functional is of course only defined for subdomains $\Omega_{k}$ such that $\Gamma_{k, c t r} \neq \emptyset$. The other subproblems simply consist of two coupled Helmholtz equation and have no control variable.

The optimality condition for the minimization of the functional $J_{k}$ can be written:

$$
J_{k}^{\prime}\left(\tilde{v}_{k}\right) \cdot\left(w_{k}-\tilde{v}_{k}\right) \geq 0, \quad \forall w_{k} \in U_{k},
$$


where $\tilde{v}_{k}$ is the optimal control.

Let us define $\delta v_{k}=w_{k}-\tilde{v}_{k}$. We denote by $\left(\delta u_{k}, \delta p_{k}\right)$, functions of $\delta v_{k}$, the solutions of the linear equations (24) (25) (27) with $\delta u_{k}, \delta p_{k}, \delta v_{k}$ instead of $u_{k}^{n+1}, p_{k}^{n+1}, v_{k}^{n+1}$ and with every source term set to 0 .

With these notations (29) may be rewritten as

$$
\begin{aligned}
& \int_{\Omega_{k}} \Re e\left(\tilde{u}_{k}, \overline{\delta u_{k}}\right) d x+\alpha \int_{\Gamma_{k, c t r}} \Re e\left(\tilde{v}_{k}, \overline{\delta v_{k}}\right) d \sigma+ \\
& \sum_{k} \lambda \int_{\Sigma_{k j}} \Re e\left(\frac{\partial}{\partial \nu_{k}} \tilde{p}_{k}, \frac{\partial}{\partial \nu_{k}} \overline{\delta p_{k}}\right)+\Re e\left(\tilde{p}_{k}, \overline{\delta p_{k}}\right) d \sigma \geq 0, \quad \forall \delta v_{k} \in U_{k}-\tilde{v}_{k} .
\end{aligned}
$$

On the other hand, using the Green formula applied to $p_{k}^{n+1}$ and $\overline{\delta v_{k}}$ and the above equations we obtain:

$$
\begin{aligned}
\int_{\Gamma_{k, c t r}}\left(p_{k}^{n+1}, \overline{\delta v_{k}}\right) d \sigma= & \int_{\Omega_{k}}\left(u_{k}^{n+1}, \overline{\delta u_{k}}\right) d x+ \\
& \sum_{k} \lambda \int_{\Sigma_{k j}}\left(\frac{\partial}{\partial \nu_{k}} p_{k}^{n+1}, \frac{\partial}{\partial \nu_{k}} \overline{\delta p_{k}}\right)+\left(p_{k}^{n+1}, \overline{\delta p_{k}}\right) d \sigma
\end{aligned}
$$

Taking the real part of the above quantity, we see that (30) (31) reduce to

$$
\int_{\Gamma_{k, c t r}} \Re e\left(\tilde{p}_{k}+\alpha \tilde{v_{k}}, \overline{w_{k}-\tilde{v}_{k}}\right) d \sigma \geq 0 \forall w_{k} \in U_{k} .
$$

We recognize (26). Therefore, $\tilde{u_{k}}, \tilde{p_{k}}, \tilde{v_{k}}$ solve the subproblem (24) (25) (27) (26). This subproblem is equivalent to the minimization of $J_{k}$ given above.

This local cost function is composed of two terms. One term is simply the restriction to the considered subdomain of the original global cost function. The other term arise because of the coupling introduced in the transmission conditions (27). It aims at minimizing the Neumann and Dirichlet boundary values of $p_{k}^{n+1}$. This makes sense as we intend $p_{k}^{n+1}$ to converge to $p$ on $\Omega_{k}$ and therefore to the smallest possible value as $p$ provide an expression of the gradient of $\int_{\Omega} \frac{1}{2}|u(v)|^{2} d x$, the scattering term of our cost function $J$.

This interpretation of is only valid for the particular form of the cost function (20) and for the subdomains on which a control is applied. In a boundary and observation control case, for example the one described in section 3.2.5 where we use the functional (38). The observation $u(v)$ will act on the subdomains bordering $\Gamma_{e} x t$ while the control will be split on the subdomains satisfying $\Gamma_{k, c t r} \neq \emptyset$. The geometrical domain decomposition may be such that these two class of subdomains have an empty intersection in which case the first term of the functional (28) disappear. It is natural to believe that such a decomposition will have some influence on the rate of convergence.

We finally note that whatever the problem, the optimization process will be restricted to the subdomains with non empty intersection with the support of the control variable. The number of degrees of freedom on which an actual optimization will be performed can therefore be greatly reduced compared to the global optimal control problem. 


\subsubsection{The convergence result}

Let $(u, p, v)$ denotes the solution of the global optimal control problem (7) (18) (21) (22). We define the errors of our approximate sequence with the exact solution by :

$$
e u_{k}^{n}=u_{k}^{n}-u, e p_{k}^{n}=p_{k}^{n}-p, e v_{k}^{n}=v_{k}^{n}-v .
$$

These errors satisfy the linear equations (24) (25) (27) (26) with $u_{i n c}=0$.

Using (22), (26) and the compatibility conditions (23), we obtain the estimate (just substract) :

$$
-\int_{\Gamma_{k, c t r}}\left|e v_{k}^{n+1}\right|^{2} d \sigma \geq \int_{\Gamma_{k, c t r}} \Re e\left(e p_{k}^{n+1} \overline{e v_{k}^{n+1}}\right) d \sigma
$$

which is to be used in the proof of convergence.

As in section 2.2.3, we define a pseudo-energy :

$$
E^{n+1}=\sum_{k \neq j} \int_{\Sigma_{k j}}\left|\frac{\partial}{\partial \nu_{k}} e u_{k}^{n+1}\right|^{2}+\lambda^{2}\left|e u_{k}^{n+1}\right|^{2}+\left|\frac{\partial}{\partial \nu_{k}} e p_{k}^{n+1}\right|^{2}+\lambda^{2}\left|e p_{k}^{n+1}\right|^{2} d \sigma .
$$

This is a natural extension of (14).

The domain decomposition algorithm decreases this 'pseudo-energy'. Indeed we have:

Proposition 2 The "pseudo-energy" satisfies

$$
\begin{aligned}
E^{n+1} \leq E^{n}-\lambda \sum_{k}( & \int_{\Omega_{k}}\left|e u_{k}^{n+1}\right|^{2} d x+\int_{\Gamma_{k, c t r}}\left|e v_{k}^{n+1}\right|^{2} d \sigma \\
& \left.+\int_{\Omega_{k}}\left|e u_{k}^{n}\right|^{2} d x+\int_{\Gamma_{k, c t r}}\left|e v_{k}^{n}\right|^{2} d \sigma\right) .
\end{aligned}
$$

Let us proceed to the proof of this proposition. Using the equation on the errors we obtain

$$
\begin{aligned}
& \Re e\left(\int_{\partial \Omega_{k}} \frac{\partial}{\partial \nu_{k}} e p_{k}^{n+1} \overline{\lambda e u_{k}^{n+1}}-\frac{\partial}{\partial \nu_{k}} e u_{k}^{n+1} \overline{\lambda e p_{k}^{n+1}} d \sigma\right) \\
= & \Re e\left(\lambda \int_{\Omega_{k}} \nabla e p_{k}^{n+1} \overline{\nabla e u_{k}^{n+1}}-\nabla e u_{k}^{n+1} \overline{\nabla e p_{k}^{n+1}}+\omega^{2}\left(e p_{k}^{n+1} \overline{e u_{k}^{n+1}}-e u_{k}^{n+1} \overline{e p_{k}^{n+1}} d x\right)\right. \\
& +\lambda \int_{\Omega_{k}}\left|e u_{k}^{n+1}\right|^{2} d x \\
= & \lambda \int_{\Omega_{k}}\left|e u_{k}^{n+1}\right|^{2} d x .
\end{aligned}
$$

Note that the terms which vanish on the right hand side of this equality precisely do because $u$ and $p$ solve adjoint equations. Using the boundary conditions on $\Gamma_{k, \text { int }}$ and $\Gamma_{k, c t r}$ and $\Gamma_{k, \text { int }}$, we replace in the left hand side of (35). The boundary terms on $\Gamma_{\text {int }}$ and $\Gamma_{\text {ext }}$ again 
vanish because of the adjointness of the the boundary conditions. This is a general feature of the algorithm which will prove useful in different situations (as we will see in section 5.2).

$$
\begin{aligned}
& \Re e\left(\sum_{j} \int_{\Sigma k, j} \frac{\partial}{\partial \nu_{k}} e p_{k}^{n+1} \overline{\lambda e u_{k}^{n+1}}-\frac{\partial}{\partial \nu_{k}} e u_{k}^{n+1} \overline{\lambda e p_{k}^{n+1}} d \sigma\right) \\
= & \Re e\left(\lambda \int_{\Gamma_{k, e x t}} i\left(e p_{k}^{n+1} \overline{e u_{k}^{n+1}}+e u_{k}^{n+1} \overline{e p_{k}^{n+1}} d \sigma\right)\right. \\
& +\lambda \int_{\Omega_{k}}\left|e u_{k}^{n+1}\right|^{2} d x+\Re e\left(\lambda \int_{\Gamma_{k, c t r}} e p_{k}^{n+1} \overline{e u_{k}^{n+1}} d \sigma\right) \\
= & \lambda \int_{\Omega_{k}}\left|e u_{k}^{n+1}\right|^{2} d x-\Re e\left(\lambda \int_{\Gamma_{k, c t r}} e p_{k}^{n+1} \overline{e v_{k}^{n+1}} d \sigma\right) .
\end{aligned}
$$

We can now rewrite the pseudo-energy

$$
\begin{aligned}
E^{n+1}= & \sum_{k \neq j} \int_{\Sigma_{k j}}\left|\frac{\partial}{\partial \nu_{k}} e u_{k}^{n+1}-\lambda e p_{k}^{n+1}\right|^{2}+\left|\frac{\partial}{\partial \nu_{k}} e p_{k}^{n+1}+\lambda e p_{k}^{n+1}\right|^{2} d \sigma+ \\
& \sum_{k}\left(\Re e\left(\lambda \int_{\Gamma_{k, c t r}} e p_{k}^{n+1} \overline{e v_{k}^{n+1}} d \sigma\right)-\lambda \int_{\Omega_{k}}\left|e u_{k}^{n+1}\right|^{2} d x\right) .
\end{aligned}
$$

The transmission conditions (27) and a equation at step $n$ similar to (36) give

$$
\begin{aligned}
E^{n+1}=E^{n}+ & \sum_{k}\left(\Re e\left(\lambda \int_{\Gamma_{k, c t r}} e p_{k}^{n+1} \overline{e v_{k}^{n+1}} d \sigma\right)-\lambda \int_{\Omega_{k}}\left|e u_{k}^{n+1}\right|^{2} d x+\right. \\
& \left.\Re e\left(\lambda \int_{\Gamma_{k, c t r}} e p_{k}^{n} \overline{e v_{k}^{n}} d \sigma\right)-\lambda \int_{\Omega_{k}}\left|e u_{k}^{n}\right|^{2} d x\right)
\end{aligned}
$$

It is time to use the optimality conditions, that is, the estimate (32). Substituting into the above expression, we finally prove (34). It can be straightforwardly deduced that (34):

i) $\left(E^{n}\right)$ is a bounded sequence.

ii) $\sum_{k} \int_{\Omega_{k}}\left|e u_{k}^{n}\right|^{2} d x$ goes to 0 as the generic term of a convergent serie.

iii) $\sum_{k} \int_{\Gamma_{k, \text { ctr }}}\left|e v_{k}^{n}\right|^{2} d \sigma$ goes to 0 as the generic term of a convergent serie.

The convergence theorem now is

Theorem 2 For all $k$, the following quantities

$$
\begin{aligned}
& \int_{\Omega_{k}}\left|\nabla u_{k}^{n}-\nabla u\right|^{2}+\left|u_{k}^{n}-u\right|^{2} d x \\
& \int_{\Omega_{k}}\left|\nabla p_{k}^{n}-\nabla p\right|^{2}+\left|p_{k}^{n}-p\right|^{2} d x \\
& \int_{\Gamma_{k, \text { ctr }}}\left|v_{k}^{n}-v\right|^{2} d \sigma
\end{aligned}
$$


go to 0 with $n$.

The proof, relying on $i$ ), ii), iii) is similar to the proof of theorem 1 detailed in [17] (see also $[3])$.

The interested (and courageous) reader will actually see by making the computations himself that the classical Robin boundary condition of section 2 cannot yield the same convergence result (because of the coupling, see remark in section 3.2.2).

Inequation (34) indicates a different behavior of the algorithm for the optimal control problem compared to (14) for the plain Helmholtz problem. The decrease of the pseudoenergy does not depend anymore on the boundary condition or even on differential operator used in the direct problem but only on the second hand term of the adjoint equation. This will prove useful in section 5.2 where we discuss different problems and boundary conditions.

\subsubsection{Minimization of the Far field}

The above method apply to a wide class of linear optimal control problems (see [27] for a review of such problems).

We focus in this section to the case of a non-local cost function involving the expression of the far field (used to define the radar cross section) which turns out to be more interesting from the application point of view. We take

$$
J(u(v), v)=\left\{\int_{A} \frac{1}{2}|C(u(v), \theta)|^{2} d \theta+\frac{\nu}{2} \int_{\Gamma_{c t r}}|v|^{2} d \sigma\right\}
$$

where, in $2-\mathrm{D}, A$ is a given subset of $[0, \pi]$ and

$$
C(u, \theta)=\frac{-i}{2 \omega^{\frac{1}{2}}} \int_{\Gamma_{\text {cont }}}\left(\frac{\partial}{\partial \nu} u(M)+i \omega(\nu \cdot \vec{d}) u(M)\right) e^{-i \omega O \vec{M} \cdot \vec{d}} d \sigma(M) .
$$

$C$ is the far field in the direction $\theta . \Gamma_{\text {cont }}$ is a contour surface containing the scattering obstacle (see figure 5), $M$ is a point running on $\Gamma_{\text {cont }}, O$ is a fixed point inside this contour and $\vec{d}=(\cos (\theta), \sin (\theta)) . u$ is given by equations (7) (18).

If we choose $\Gamma_{\text {cont }}=\Gamma_{\text {ext }}$ and if this absorbing boundary is far enough to the obstacle with respect to the wave length, an approximation of the cost function is simply

$$
J(u(v), v)=\left\{\int_{\Gamma_{e x t}} \frac{1}{2} \mid\left(\left.u(v)\right|^{2} d \sigma+\frac{\nu}{2} \int_{\Gamma_{c t r}}|v|^{2} d \sigma\right\}\right.
$$

The adjoint equation, analog of (21), is

$$
\left\{\begin{array}{lr}
-\Delta p-\omega^{2} p=0 & \text { in } \Omega \\
\frac{\partial}{\partial y} p=0 & \text { on } \Gamma_{\text {int }} \cup \Gamma_{c t r} \\
\frac{\partial}{\partial \nu} p-i \omega p=u & \text { on } \Gamma_{\text {ext }}
\end{array}\right.
$$

$\mathrm{RR} \mathrm{n}^{\circ} 2791$ 
The optimality condition (22) is unchanged and the domain decomposition and proof of convergence easily adapted.

If we decide to keep the exact formulation of the far field as given in (37), the adjoint equation is now

$$
\left\{\begin{array}{lr}
-\Delta p-\omega^{2} p=C^{*}(C(u)) & \text { in } \Omega \\
\frac{\partial}{\partial y} p=0 & \text { on } \Gamma_{i n t} \cup \Gamma_{c t r} \\
\frac{\partial}{\partial \nu} p-i \omega p=0 & \text { on } \Gamma_{\text {ext }}
\end{array}\right.
$$

where $C^{*}$ is the adjoint operator of $C$ and the optimality condition (22) is still unchanged. A mathematical study of existence and uniqueness of solutions for such a problem can be found in [22].

We now discuss the application of our algorithm to this problem. We modify accordingly our algorithm and (25) becomes

$$
-\Delta p_{k}^{n+1}-\omega^{2} p_{k}^{n+1}=C^{*}\left(C\left(u_{c o n t}^{n+1}\right)\right) \text { in } \Omega_{k}
$$

Where for all $k, u_{\text {cont }}^{n+1}=u_{k}^{n+1}$ on $\Gamma_{k, \text { cont }}$ with the evident notation $\Gamma_{k, \text { cont }}=\partial \Omega_{k} \cap \Gamma_{\text {cont }}$ (see figure 5).

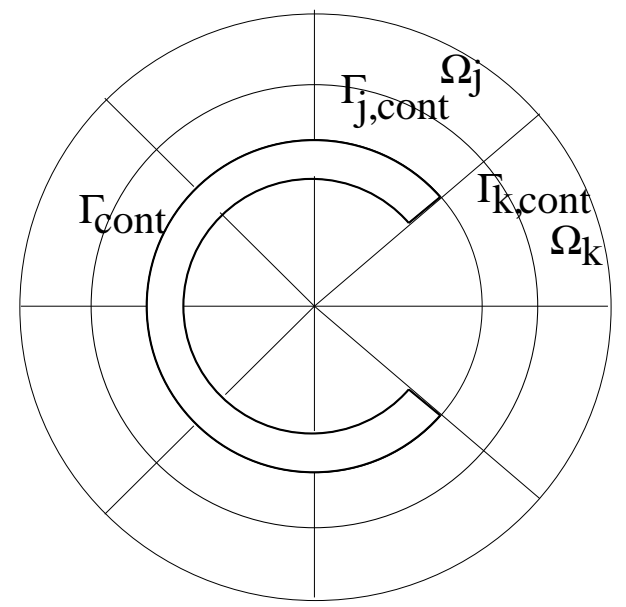

Figure 5: decomposition of the contour

Convergence of the algorithm with the same coupled transmissions condition (27) can be proved using the same kind of arguments.

Nevertheless due to the non local character of the functional $C$ we see that the resolution of the subproblems set in subdomains having non empty intersection with $\Gamma_{\text {cont }}$ is non longer explicit but are coupled through the right hand side of equation (39). A simple solution to 
this problem is of course to decompose the domain $\Omega$ such that $\Gamma_{\text {cont }}$ be fully contained in only one subdomain.

One could also try to relax the coupling term by replacing it by $C^{*}\left(C\left(u_{\text {cont }}^{n}\right)\right)$ which is available from the previous iteration. In this case, we cannot prove convergence.

\section{Variants of the algorithm}

We present two variants of the algorithm for the direct scattering problem. One is a simple modification of the transmission condition, the other uses an 'optimal control' like algorithm. They will be used to solve more general problem in section 5.2.

\subsection{Under-relaxation}

A slight modification of the basic algorithm generates a new algorithm with which has a much better rate of convergence in applications [18]. We call it the under-relaxed algorithm because of the introduction of a real parameter $r \in$ ] $0 ., 0.5$ [ which may be viewed as a relaxation parameter.

For the direct problem it simply consists in the following modification of the transmission condition in (12) :

$$
\frac{\partial}{\partial \nu_{k}} u_{k}^{n+1}+i \omega u_{k}^{n+1}=(1-r)\left(-\frac{\partial}{\partial \nu_{j}} u_{j}^{n}+i \omega u_{j}^{n}\right)+r\left(\frac{\partial}{\partial \nu_{k}} u_{k}^{n}+i \omega u_{k}^{n}\right) \text { on } \Sigma_{k j}
$$

We find (after some computations) that the new law for the decrease of the "pseudoenergy" (13) is now given by

$$
\begin{aligned}
E^{n+1}=E^{n} & -2 \omega^{2}\left(\sum_{k} \int_{\Gamma_{k, e x t}}\left|e u_{k}^{n+1}\right|^{2} d \sigma-(1-2 r) \int_{\Gamma_{k, e x t}}\left|e u_{k}^{n}\right|^{2} d \sigma\right) \\
& -2 r(1-r)\left(\sum_{k, j} \int_{\Sigma_{k j}}\left|\frac{\partial}{\partial \nu_{k}} e u_{k}^{n}+\frac{\partial}{\partial \nu_{j}} e u_{j}^{n}\right|^{2}+\omega^{2}\left|e u_{k}^{n}-e u_{j}^{n}\right|^{2} d \sigma\right) .
\end{aligned}
$$

In addition to the usual norm of the error on the external boundaries $\Gamma_{k, e x t}$ (first line of (41)), the "pseudo-energy" is decreased by a new factor which depends on the relaxation parameter (second line). This new quantity turns out to be a norm of the error ([17]). Indeed, if this term is null, the error satisfies an Helmholtz equation on the whole domain with homogeneous boundary condition. This implies that the error is zero everywhere.

The same modification of the transmission condition and remark on the behavior of the under-relaxed algorithm also hold for the optimal control case.

$\mathrm{RR} \mathbf{n}^{\circ} 2791$ 
Jean-David Benamou , Bruno Desprès

\subsection{Using the optimal control algorithm to solve the plain direct problem}

In this section we explain how the 'Optimal Control' algorithm can be used to derive to solve the plain direct Helmholtz problem.

We first remark that we can add a fictive adjoint problem to the direct scattering problem. Instead of solving simply (7) we also consider the problem

$$
\left\{\begin{array}{lr}
-\Delta p-\omega^{2} p=u & \text { in } \Omega \\
\frac{\partial}{\partial y} p=0 & \text { on } \Gamma_{\text {int }} \cup \Gamma_{c t r} \\
\frac{\partial}{\partial \nu} p-i \omega p=0 & \text { on } \Gamma_{\text {ext }}
\end{array}\right.
$$

This 'adjoint' problem is well posed and depends on $u$. It is similar to the optimal control problem of section 4 except for the absence of control variable $v$ and optimality condition $(22)$.

We now apply the domain decomposition (24) (25) (27) of section 4 (where we 'forget' the control variable $v_{k}^{n+1}$ and the optimality condition (26)) to solve the coupled problem (7) (42).

The proof of convergence is similar to and actually simpler than the proof of section 3.2.4 (there are no optimality conditions (22), (26) and hence no estimate (32). The law of decrease for the pseudo-energy is still given by (33) :

$$
E^{n+1}=E^{n}-\lambda \sum_{k}\left(\int_{\Omega_{k}}\left|e u_{k}^{n}+1\right|^{2}+\left|e u_{k}^{n}\right|^{2} d x\right) .
$$

\section{Solving other problems}

\subsection{The inhomogeneous case}

We now come back to the inhomogeneous problem (1) with non-constant coefficients $\mu$ and $\rho$. These coefficients are supposed to be piecewise $C^{1}$, so that the problem is well posed.

We modify the domain decomposition algorithm as follows

$$
\left\{\begin{array}{lr}
-\nabla\left(\mu_{k} \vec{\nabla} u_{k}^{n+1}\right)-\omega^{2} \rho u_{k}^{n+1}=0 & \text { in } \Omega_{k} \\
\mu_{k} \frac{\partial}{\partial \nu_{k}} u_{k}^{n+1}+i \beta_{k} \omega u_{k}^{n+1}=-\mu_{k} \frac{\partial}{\partial \nu_{j}} u_{j}^{n}+i \beta_{j} \omega u_{j}^{n} & \text { on } \Sigma_{k j} \\
\mu_{k} \frac{\partial}{\partial \nu_{k}} u_{k}^{n+1}+i \beta_{k} \omega u_{k}^{n+1}=0 & \text { on } \Gamma_{k, e x t} \\
\frac{\partial}{\partial \nu_{k}} u_{k}^{n+1}=\frac{\partial}{\partial \nu_{k}} u_{i n c} & \text { on } \Gamma_{k, i n t .} .
\end{array}\right.
$$

$\mu_{k}$ denote the value of $\mu$ in $\Omega_{k}$. We necessarily have $\mu_{k}=\mu_{j}$ on $\Sigma_{k j}$. The $\left(\beta_{k}\right)$ s are real positive coefficients to be determined. They must also satisfy $\beta_{k}=\beta_{j}$. 
A study of the dimensionality of $\beta$ indicates that $\beta$ has to be similar to $\sqrt{\mu \rho}$. A possible choice for $\beta_{k}$ (and $\beta_{j}$ ) on $\Sigma_{k j}$ is

$$
\beta_{k}=\frac{1}{2}\left(\sqrt{\mu_{k} \rho_{k}}+\sqrt{\mu_{j} \rho_{j}}\right)
$$

the arithmetic mean value of $\sqrt{\mu_{k} \rho_{k}}$ on the interface.

The pseudo-energy has to be modified accordingly

$$
\begin{aligned}
E^{n}= & \sum_{k \neq j} \int_{\Sigma_{k j}}\left(\frac{1}{\beta_{k}}\left|\mu_{k} \frac{\partial}{\partial \nu_{k}} e u_{k}^{n}\right|^{2}+\beta_{k} \omega^{2}\left|e u_{k}^{n}\right|^{2}\right) d \sigma+ \\
& \sum_{k} \int_{\partial \Gamma_{k, e x t}}\left(\frac{1}{\beta_{k}}\left|\frac{\partial}{\partial \nu_{k}} e u_{k}^{n}\right|^{2}+\beta_{k} \omega^{2}\left|e u_{k}^{n}\right|^{2}\right) d \sigma
\end{aligned}
$$

The proof of convergence now follows the same steps as in section 2.

The optimal control case generalizes likewise to to inhomogeneous case.

\subsection{Other boundary conditions}

We already pointed out the importance of the first order absorbing condition in the convergence process (section 2.2.3). This section explains how to deal with different boundary conditions.

\subsubsection{Waveguide transparent condition}

We present here a wave guide problem which involves a non local transparent boundary condition. For more details and comments on this formulation and more general cases see [32]. The wave guide is made of an infinite 2 -D stripe $\Omega$ defined by $0<y<L$ in a space described with cartesian coordinate $(x, y)$ (see figure 6)

If we consider the homogeneous Helmholtz equation (7) inside $\Omega$ and Neumann boundary conditions $\frac{\partial}{\partial y} u=0$ on $y=0, L$, the solution $u$ can be decomposed on an infinite number of modes. More precisely we write

$$
u(x, y)=\sum_{l=0, \infty} \hat{u}_{l}(x) \cos \left(\frac{l \pi}{L} y\right)
$$

The lieth mode $\hat{u}$ satisfies the 1-D Helmholtz equation (we normalize $L$ to 1 for simplicity)

$$
-\frac{\partial^{2}}{\partial x^{2}} \hat{u}_{l}+\left(l^{2} \pi^{2}-\omega^{2}\right) \hat{u}_{l}=0
$$

for which an exact analytic solution can be derived.

$\mathrm{RR} \mathbf{n}^{\circ} 2791$ 


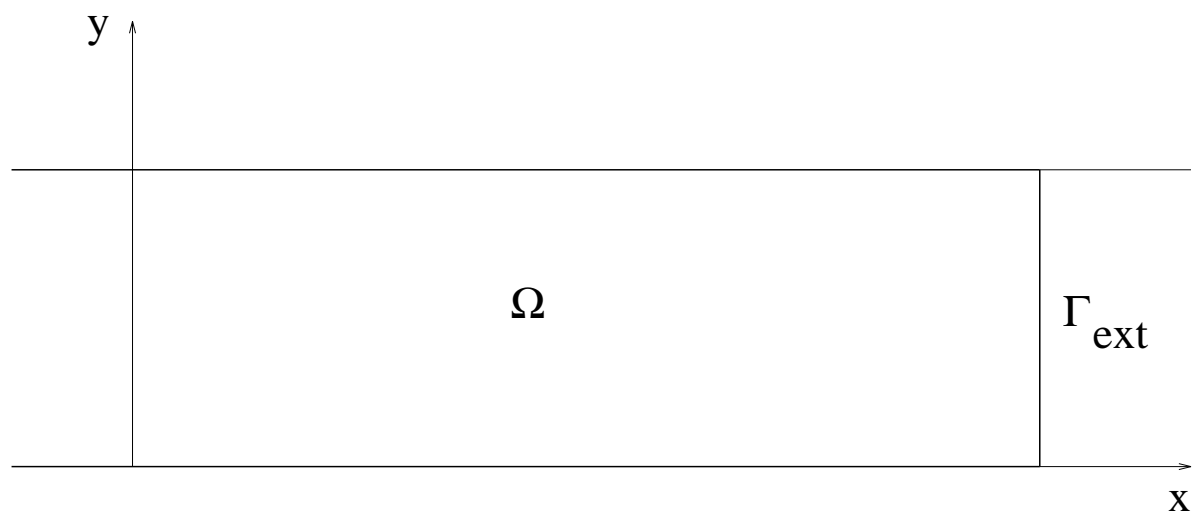

Figure 6: A wave guide.

If we decide to close the exit of the wave guide (on the side $x>0$ ) by a transparent boundary $\Gamma_{e x t}$ (see figure 6 ), we can derive an exact transparent boundary condition mode by mode. We have two possibilities:

First, if $l \pi<\omega$, the mode is propagative. We know the exact form of the outgoing solution of (45). It satisfies

$$
\frac{\partial}{\partial x} \hat{u}_{l}+\mathrm{i} \sqrt{\omega^{2}-l^{2} \pi^{2}} \hat{u}_{l}=0 \text { on } \Gamma_{e x t} .
$$

Secondly, if $l \pi>\omega$, the mode is evanescent and the boundary condition is

$$
\frac{\partial}{\partial x} \hat{u}_{l}+\sqrt{l^{2} \pi^{2}-\omega^{2}} \hat{u}_{l}=0 \text { on } \Gamma_{e x t} .
$$

An analogous transparent boundary condition can be defined on the $x<0$ side. If we decide to decompose the waveguide in successive slices in $x$ (figure 7 )

we can try to apply the domain decomposition method (section 2) on this example. The modal decomposition of the transmission condition (see (12)) is

$$
\frac{\partial}{\partial x} \hat{u}_{l, k}^{n+1}+\mathrm{i} \omega \hat{u}_{l, k}^{n+1}=\frac{\partial}{\partial x} \hat{u}_{l, j}^{n}+\mathrm{i} \omega \hat{u}_{l, j}^{n} \text { on } \Sigma_{k j} .
$$

We immediately see on the proof of convergence that the boundary condition (46) for propagative modes will pose no problem as this boundary condition has the form of a first order absorbing boundary condition. Indeed the corresponding pseudo-energy for the lieth mode satisfy the decrease law

$$
\hat{E}_{l}^{n+1}=\hat{E}_{l}^{n}-2 \omega^{2} \sum_{k} \int_{\Gamma_{k, e x t}}\left|\hat{e}_{l, k}^{n+1}\right|^{2}+\left|\hat{e}_{l, k}^{n}\right|^{2} d \sigma .
$$




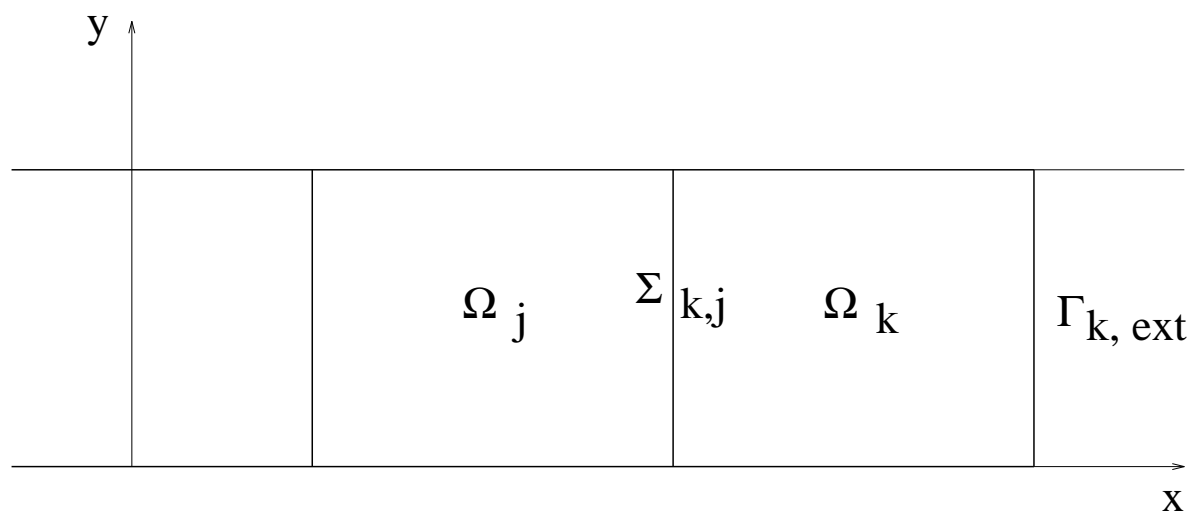

Figure 7: Decomposition of the wave guide.

Conversely, for the evanescent modes and because of the real coefficient $\sqrt{l^{2} \pi^{2}-\omega^{2}}$ in (47), the pseudo energy is stationary :

$$
\hat{E}_{l}^{n+1}=\hat{E}_{l}^{n} .
$$

The plain domain decomposition of section 2 does not converge for evanescent modes. The variants of the algorithm described in previous section can remedy to this situation.

If we use an under-relaxed transmission condition (section 4.1) i.e.

$$
\frac{\partial}{\partial \nu_{k}} \hat{u}_{l, k}^{n+1}+\mathrm{i} \omega \hat{u}_{l, k}^{n+1}=(1-r)\left(-\frac{\partial}{\partial \nu_{j}} \hat{u}_{l, j}^{n}+\mathrm{i} \omega \hat{u}_{l, j}^{n}\right)+r\left(\frac{\partial}{\partial \nu_{k}} \hat{u}_{l, k}^{n}+\mathrm{i} \omega \hat{u}_{l, k}^{n}\right),
$$

the decrease law for the pseudo-energy of the evanescent mode $\hat{E}_{l}^{n}$ is a modification of (41) :

$$
\hat{E}_{l}^{n+1}=\hat{E}_{l}^{n}-2 r(1-r)\left(\sum_{k, j} \int_{\Sigma_{k j}}\left|\frac{\partial}{\partial \nu_{k}} \hat{e}_{l, k}^{n}+\frac{\partial}{\partial \nu_{j}} \hat{e}_{l, j}^{n}\right|^{2}+\omega^{2}\left|\hat{e}_{l, k}^{n}-\hat{e}_{l, j}^{n}\right|^{2} d \sigma .\right)
$$

There are no terms supported by $\Gamma_{e x t}$ because of the evanescent boundary condition (47) but the additional terms induced by the under-relaxation guarantee convergence.

We can also use the 'optimal control' variant of section 4.2. The fictive adjoint subproblem associated to (45) (47) is (the problem is actually auto adjoint)

$$
\begin{gathered}
-\frac{\partial^{2}}{\partial x^{2}} \hat{p}_{l, k}^{n+1}+\left(l^{2} \pi^{2}-\omega^{2}\right) \hat{p}_{l, k}^{n+1}=\hat{u}_{l, k}^{n+1} \text { on } \Omega_{k}, \\
\frac{\partial}{\partial x} \hat{p}_{l, k}^{n+1}+\sqrt{l^{2} \pi^{2}-\omega^{2}} p_{l, k}^{\hat{n+1}}=0 \text { on } \Gamma_{\text {ext }} .
\end{gathered}
$$


We then add the modal decomposition of the coupled transmission conditions (27) :

$$
\begin{aligned}
& \frac{\partial}{\partial \nu_{k}} \hat{u}_{l, k}^{n+1}+\lambda \hat{p}_{l, k}^{n+1}=-\frac{\partial}{\partial \nu_{j}} \hat{u}_{l, j}^{n}+\lambda \hat{p}_{l, j}^{n} \text { on } \Sigma_{k j}, \\
& \frac{\partial}{\partial \nu_{k}} \hat{p}_{l, k}^{n+1}-\lambda \hat{u}_{l, k}^{n+1}=-\frac{\partial}{\partial \nu_{j}} \hat{p}_{l, j}^{n}-\lambda \hat{u}_{l, j}^{n} \text { on } \sigma_{k j} .
\end{aligned}
$$

This procedure converges as outlined in section 4.2 and the decrease of the pseudo-energy defined as

$$
\hat{E}_{l}^{n+1}=\sum_{k \neq j} \int_{\Sigma_{k j}}\left|\frac{\partial}{\partial \nu_{k}} e \hat{u}_{l, k}^{n+1}\right|^{2}+\lambda^{2}\left|\hat{e} \hat{u}_{l, k}^{n+1}\right|^{2}+\left|\frac{\partial}{\partial \nu_{k}} \hat{e p}_{l, k}^{n+1}\right|^{2}+\lambda^{2}\left|\hat{e} \hat{p}_{l, k}^{n+1}\right|^{2} d \sigma .
$$

is given by (34).

The introduction of a fictive adjoint problem allows to deal with the embarrassing terms on the boundary (as well as the non coercive terms in the Helmholtz equation) and add a coercive term on the right hand side of (48) which will guarantee the decrease of the pseudo energy and hence the convergence.

The arguments developed in the section also hold for other choice of non local boundary condition on $\Gamma_{e x t}$ such as a second order absorbing boundary condition (4).

\subsubsection{PML absorbing layers}

In the case of PML (6) the absorbing layer replaces in some sense the absorbing boundary conditions. Let us note that, unlike for classical absorbing boundary conditions, it seems no trivial to prove existence of a solution to the system with PML layers. So in this section we simply postulate the existence of the solution with a PML layer.

We now go a bit faster and simply examine the behavior the algorithm on subdomains consisting of infinite vertical stripes. This will formally be enough to point out convergence failures and possible cures.

The subdomains are infinite stripes in the $x$ direction. The interfaces $\Sigma_{k j}$ are now lines of equations $x=c s t$.. We assume that $d$ is constant on the subdomains contained in the absorbing layer. For such a subdomain the pseudo energy has to be modified (as in the inhomogeneous case) and uses terms of the form

$$
\int_{\Sigma_{k j}}\left(\left|d \frac{\partial}{\partial y} e u_{k}^{n}\right|^{2}+\omega^{2}\left|e u_{k}^{n}\right|^{2}\right) d \sigma
$$

The key point of the demonstration (section 2.2.3) with the simple transmission condition (see (12)) is to evaluate the cross products

$$
\Re e\left(\int_{\partial \Omega_{k}} d \frac{\partial}{\partial \nu_{k}} e u_{k}^{n+1} \overline{i \omega e u_{k}^{n+1}} d \sigma\right)
$$


which expresses the quantity which decreases the pseudo-energy. We integrate by part an equation of the type (6) for the error and obtain an equivalent of (16) :

$$
\begin{gathered}
\Re e\left(\int_{\partial \Omega_{k}} d \frac{\partial}{\partial \nu_{k}} e u_{k}^{n+1} \overline{i \omega e u_{k}^{n+1}} d \sigma\right) \\
=\Re e\left(-i \omega\left(\int_{\Omega_{k}} \frac{1}{d}\left|\frac{\partial}{\partial x} e u_{k}^{n+1}\right|^{2}+d\left|\frac{\partial}{\partial y} e u_{k}^{n+1}\right|^{2}-\frac{\omega^{2}}{d}\left|e u_{k}^{n+1}\right|^{2}\right) d x\right)
\end{gathered}
$$

In (16) the right hand side was vanishing. Now $d$ is a complex number and we immediately see that the real part on these different coefficients are going to be of opposite sign.

It is therefore not possible to prove the convergence of the classical domain decomposition method. Nor does it seem trivial to use an under-relaxed variant of the algorithm or more general transmission conditions with an arbitrary complex parameter instead of the pure imaginary $i \omega$. Conversely, the 'optimal control' algorithm can solve the problem.

As in section 5.2.1 we define a fictive adjoint of equation (6). As $d$ is a complex parameter we consider

$$
-\partial_{x x}^{2} p-\bar{d} \partial_{y}\left(\bar{d} \partial_{y} p\right)-\omega^{2} p=u
$$

then the domain decomposition described in section 4.2 is easily applied to solve (6) (50) and the proof of convergence gives the same law of decrease for the pseudo-energy.

\section{Numerical resolution}

\subsection{Discrete formulation and Implementation}

In the framework of the domain decomposition method, various strategies are possible with regard to the shape and number of subdomains, discretization and method of resolution of the subproblems.

It is possible to work on a discrete formulation of the global problem. Mixed hybrid finite elements (see [21] [31] on this technique) are for instance well suited to our algorithm for it uses in particular, as degrees of freedom, the fluxes of the normal derivative and the average values of the trace of the direct and adjoint states on the interfaces which are the natural unknowns of our transmission conditions. It allows in particular a direct transcription of the domain decomposition algorithm and the proof of its convergence to the discrete formulation.

A massively parallel strategy has been implemented on a Connection Machine (see [4] [5]). In this approach each finite element is taken as one subdomain. Thank to the small number of degrees of freedom in each subdomain, the subproblems can then be solved an-

alytically beforehand. The algorithm then reduces to explicit formulae and transmission of data between subdomains. We were able to solve an optimal control problem for the 3-D Helmholtz equation with about $510^{5}$ tetrahedrons in less than 4 hours on a $16 \mathrm{k}$ CM200.

$\mathrm{RR} \mathbf{n}^{\circ} 2791$ 
Jean-David Benamou , Bruno Desprès

The memory limit of this machine would allow to treat optimal control problems up to $1.510^{6}$ finite elements.

We emphasize that all the material presented in this paper can be generalized to the Maxwell's equation in the frequency domain.

We applied these techniques [20] on a parallel CRAY T3D computer to solve the direct 3-D Maxwell's equations. Without going into the details, we mention that it was possible to take into account more than $1510^{6}$ tetrahedrons in the finite element approximation and to solve the system in less than 1 hour. The domain decomposition was coarse and a conjugate gradient algorithm was used for the resolution of the subproblems.

\subsection{A numerical experiment}

We illustrate this paper by the resolution of the problem described in section 2 for the direct problem and section 3 for the optimal control problem. We consider the unconstrained case, i.e. the control $v$ is free to take any value. A plane wave arrives from the right. Top of figure 8 , there is no control, we see the multiple reflection caused by the hard resonator. At the bottom the optimal control solution generated by our algorithm. Reflections are 'killed' inside but not outside as the control only acts on the inside boundary of the resonator. Small oscillations persist inside of the resonator. Two possible explanations for this phenomenon are : first the edges of the resonator which still scatter the incoming wave, secondly the penalization term in the cost function which takes into account the energy of the control and therefore acts as a constraint on it.

\section{Conclusion}

This method has motivated several studies and extensions.

It was extended to the Maxwell equations in [19] and used for 3-D computation of electromagnetic scattered fields. Non local transmission operators have been studied in [35]. A 'Gauss-Seidel' variant of the transmission condition has been studied in [29] for the Maxwell equations [20]. The method has inspired more sophisticated numerical algorithm such as conjugate gradient like iterative algorithm [14] [18] and a new weak formulation of the Helmholtz equation [11].

The method is easily applied to dispersive media [25] and coercive elliptic equations [17] [26] [3]

Let us finally mention the existence of a related work in [38].

The extension to optimal control problem described in this paper has been used to solve 3-D acoustic problems [5]. Its application to electromagnetic active control is under investigation.

The application of these techniques to the computation of electromagnetic waveguide is also studied. 

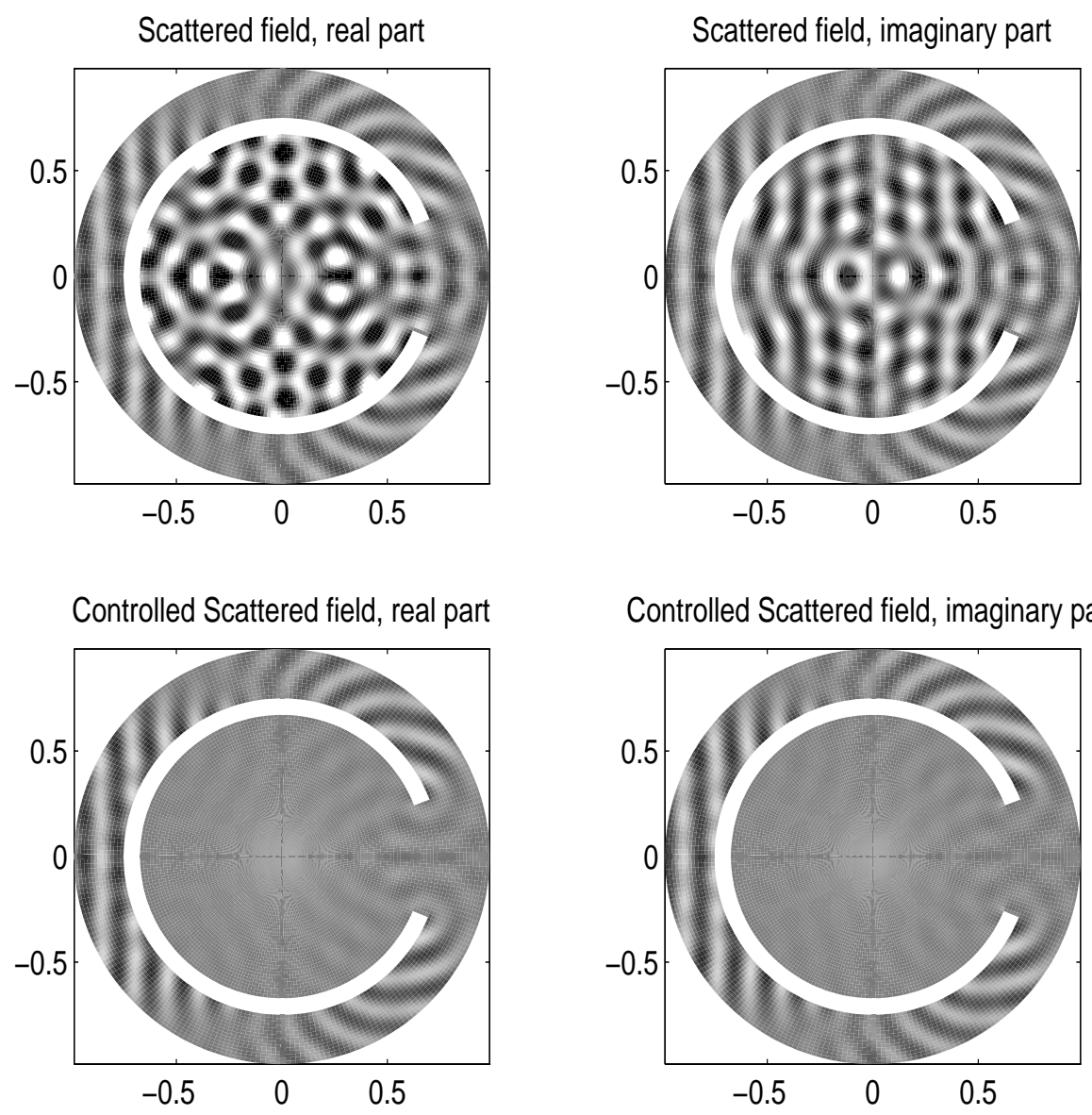

Figure 8: Scattered field, a plane wave arrives from the right.

\section{References}

[1] C. Atamian, Q.V. Dinh, R. Glowinsky, J. He, and J. Periaux. Control approach to fictitious-domain methods. Application to fluid-dynamics and electro-magnetics. In SIAM, editor, Fourth international symposium on domain decomposition method for partial differential equations, pages 275-309, 1991.

[2] A. Bayliss and E. Turkel. Radiation boundary conditions for wave like equations. Comm. Pure and Appl. Math., 33:707-725, 1980. 
[3] J.D. Benamou. Domain decomposition methods with coupled transmission conditions for the optimal control of systems governed by elliptic partial differential equations. to appear in SINUM.

[4] J.D. Benamou. A domain decomposition method for the optimal control of system governed by the helmholtz equation. In SIAM, editor, Third international conference on mathematical and numerical wave propagation phenomena (Cannes-Mandelieu), 1995.

[5] J.D. Benamou. A massively parallel algorithm for the optimal control of systems governed by elliptic p.d.e.'s. In SIAM, editor, Seventh SIAM conference on parallel processing for scientific computing (San Francisco), 1995.

[6] J. Berenger. A perfectly matched layer for the absortion of electromagnetic waves. $J$. Comp. Phys., 114:185-200, 1994.

[7] A. Bespalov. Application of fictitious domain method to the solution of the helmholtz equation in unbounded domain. Rapport INRIA RR-1797, 1992.

[8] D. Bouche and F. Molinet. Méthodes asymptotiques en électromagnétisme. Masson, 1989.

[9] X.-C. Cai and O. B. Widlund. Domain decomposition algorithm for indefinite elliptic problems. Siam J. Sci. Stat. Comput., 13:243-258, 1992.

[10] X.-C. Cai and O. B. Widlund. Multiplicative algorithms for some nomsymmetric and indefinite problems. Siam J. Numer. Anal.., 30:936-952, 1993.

[11] O. Cessenat and B. Despres. Une nouvelle formulation variationnelle des équations d'ondes en fréquence. application au problème de Helmholtz 2d. Technical Report 2779, CEA, 1994.

[12] F. Clement, M. Kern, and C. Rubin. Solution of the 3D Helmholtz equation by conjugate gradients. In Copper mountain conference on iterative methods, 1990.

[13] D. Colton and R. Kress. Integral equation method in scattering. Wiley-Interscience, 1983.

[14] A. de la Bourdonnaye. A substructuring method for a harmonic wave propagating problem : ananlysis of the conditioning number of the problem on the interfaces. Technical Report 95-35, CERMICS, 1995.

[15] B. Després. A non-overlapping linear domain decomposition method for the helmholtz problem. submitted to SINUM.

[16] B. Després. Domain decomposition method and the helmholtz problem. In SIAM, editor, Mathematical and Numerical Aspects of Wave Propagation Phenomena, pages 44-52, 1991. 
[17] B. Després. Méthodes de décomposition de domaine pour les problèmes de propagation d'ondes en régimes harmoniques. PhD thesis, Paris 9 France, 1991.

[18] B. Després. Domain decomposition method and the Helmholtz problem (part ii). In Second international conference on mathematical and numerical aspects of wave propagation phenoma. SIAM, 1993.

[19] B. Després, P. Joly, and J. E. Roberts. A domain decomposition method for the harmonic maxwell's equations. In IMACS international symposium on iterative methods in linear algebra. North Holland, 1990.

[20] Bruno Despres. Implementation of a non overlapping domain decomposition method on a cray t3d, for solving the $3 \mathrm{~d}$ harmonic maxwell's equations. 1994. IMA international symposium on Maxwell's equations.

[21] J. E. Roberts G. Chavent. A unified physical presentation of mixed, mixed-hybrid finite elements and usual finite differences for the determination of velocities in waterflow problems. To appear in Advances Water Resources.

[22] J. Paoli G. Roge, C. Bardos. Maîtrise du champ rétrodiffusé par une méthode de contôle optimal. submitted to sinum.

[23] D. Givoli. Non reflecting boundary conditions. J. Comp. Phys., 94:1-29, 1991.

[24] C. I. Goldstein. The finite element method with non uniform mesh sizes applied to the exterior Helmholtz problem. Num. Math., 38:61-82, 1981.

[25] J. E. Santos J. Douglas, F. Pereira. A parallelizable approach to the simulation of waves in dispersive media. In SIAM, editor, Third international conference on mathematical and numerical wave propagation phenomena (Cannes-Mandelieu), 1995.

[26] J. E. Roberts J. Wang J. Douglas Jr., P.J. Paes Leme. A parallel iterative procedure applicable to the approximate solution of second order partial differential equations by mixed finite element methods. Numer. Math., 65:95-108, 1993.

[27] J.L. Lions. Contrôle optimal de systèmes gouvernés par des équations aux dérivées partielles. Dunod, Paris, 1968.

[28] P.L. Lions. On the schwarz alternating method 3. In SIAM, editor, Third international symposium on domain decomposition methods for partial differential equations, 1990.

[29] P.Joly M. Invernizzi, F. Collino and A. Piacentini. A new transmission operator in the domain decomposition for the maxwell equations. In ECNUM, 1995.

[30] J. C. Nedelec. Curved finite element methods for the solution of singular integral equations on surfaces on $R^{n}$. Comp. Meth. in Appl. Mech. and Eng., 8:61-80, 1971.

RR $n^{\circ} 2791$ 
[31] J. C. Nedelec. A new family of mixed finite elements in $r^{3}$. Num. Math., 50:57-81, 1986.

[32] C. Poirier P. Joly. Mathematical analysis of electromagnetic open waveguides. M2AN, 29:505-575, 1995 .

[33] J.L. Lions R. Dautray. Analyse Mathématique et Calcul Numérique pour les Sciences et Techniques, Tome 1. Masson, Paris, 1985.

[34] C. M. Rappaport. Perfectly matched absorbing boundary conditions based on anisotropic lossy mapping of space. IEEE Microwave and Guided Wave Letters, 5, 1995.

[35] P.Joly S. Ghanemi, F. Collino. Domain decomposition method for harmonic wave equations. In SIAM, editor, Third international conference on mathematical and numerical wave propagation phenomena (Cannes-Mandelieu), 1995.

[36] Y. Saad and M.H. Schultz. Gmres : a generalized minimal algorithm for solving non symmetric linear system. SIAM J. Sci. Statiiiiist. Comput., 7:856-869, 1986.

[37] J. A. Stratton. Electromagnetic theory. McGraw-Hill, 1941.

[38] E.I. Ogorodnikov V.V. Shaidurov. Some numerical method of solving helmholtz wave equation. In SIAM, editor, Mathematical and Numerical Aspects of Wave Propagation Phenomena, pages 73-79, 1991. 


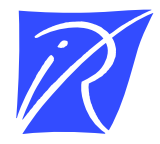

Unité de recherche INRIA Lorraine, Technopôle de Nancy-Brabois, Campus scientiøque,

615 rue du Jardin Botanique, BP 101, 54600 VILLERS LÈS NANCY

Unité de recherche INRIA Rennes, Irisa, Campus universitaire de Beaulieu, 35042 RENNES Cedex

Unité de recherche INRIA Rhône-Alpes, 46 avenue Félix Viallet, 38031 GRENOBLE Cedex 1

Unité de recherche INRIA Rocquencourt, Domaine de Voluceau, Rocquencourt, BP 105, 78153 LE CHESNAY Cedex

Unité de recherche INRIA Sophia-Antipolis, 2004 route des Lucioles, BP 93, 06902 SOPHIA-ANTIPOLIS Cedex

Éditeur

INRIA, Domaine de Voluceau, Rocquencourt, BP 105, 78153 LE CHESNAY Cedex (France)

ISSN 0249-6399 